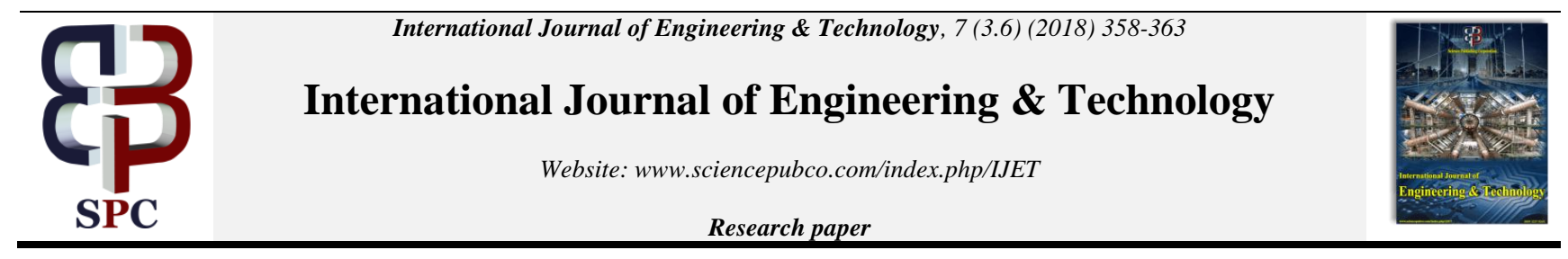

\title{
Hierarchical Honey Bee Model to Avoid Sleep Deprivation Attack in MANET
}

\author{
S. Madhurikkha ${ }^{{ }^{*}}$, R. Sabitha ${ }^{2}$ \\ ${ }^{1}$ Research Scholar, Sathyabama University, Chennai, India. \\ ${ }^{2}$ Professor, Department Of Information Technology, Jeppiaar Engineering College, Chennai, India. \\ E-Mail:Sabisam73@Gmail.Com \\ *Corresponding Author E-Mail:Madhurikkha@Gmail.Com
}

\begin{abstract}
Ad-hoc networks are highly vulnerable to the attacks due to the dynamic nature of the network topology, lack of centralized management and cooperative algorithm. Nodes in the ad-hoc network are responsible to transmit packets to other nodes. One of many different kinds of attacks, a node may experience the exhaustion of power by consuming its batter power. Routing protocol controls the packet transmission over the network and calculates the routing path for transmission. The predefined static routed path is limited in ad-hoc network, and nodes have limited energy that is depleted due to transmission. Sleep deprivation attack constantly drops the power source on a particular node until the node gets shutdown. We have proposed an effective model named Honey-bee model to enhance Ad-hoc On-Demand Distance Vector Routing algorithm. Hierarchical clustering method is used for the formation of the cluster of nodes. Based on this approach, each node is assigned with a cluster that prevents the attack. We have simulated in ns 2 tool compared results with other on-demand routing protocol such as Power Aware Routing Protocol, Dynamic Source Routing, Flow-State extensions to Dynamic Source Routing and AODV.
\end{abstract}

Keywords: On-demand routing, sleep deprivation, hierarchical clustering, routing protocol.

\section{Introduction}

Mobile Ad Hoc Networks (MANET) [1] consists of a cluster of mobile nodes that can communicate with each other and can move anywhere without any restriction. Each node in the network itself acts as a router for forwarding and receiving packets to the other nodes. Many routing protocol is designed to accomplish the task by effectively handling the routing path such as AODV, Power Aware Routing Protocol, Dynamic Source Routing, Flow-State extensions to Dynamic Source Routing. Routing in ad-hoc networks has been a challenging task due to the constant change in the network. Routing protocol determines the router's communication link between nodes. Routing algorithm specifies the direction and link of nodes that are used to transfer packets.

Nodes in the ad-hoc networks have limited power and memory source. These characteristics demand an efficient protocol to handle power consumption along with the other techniques. Target of the intruder is to maximize the power consumption of nodes, so that their lifetime is minimized. This type of attack is known as sleep deprivation attack. Routing protocol cannot determine the attacks but can efficiently handle the request to prevent power exhaustion to particular node.

AODV protocol initiates the path discovery process to identify the routing path from source node to destination node. Every node maintains two separate counters of the sequence number, and a broadcast id. The source node initiates path discovery by broadcasting a route request RREQ packet to its neighbour node. Neighbour node processes the request and sends the path to the source node if it finds the route in the routing table. Else the request is forwarded to the next node. When the path is identified, RREQ-ACK is triggered to the source node with routing path. Source starts transmitting the packets through the routing path, and the process continues if any error occurs during the transmission. Our proposed enhanced protocol prevents the sleep deprivation attack and efficiently distributes the power consumption over the nodes [2].

\section{Nomenclature}

$\mathrm{N}$ : Mobile node in the network.

Rp: Remaining power of the mobile node available for transmission.

$\mathrm{Cn}$ : Total number of mobile nodes grouped to form a colony

$\mathrm{Cp}$ : Total power available in the colony.

$C_{p}=\sum_{1}^{N} R_{p}$

$\mathrm{Tp}$ : Threshold power of the mobile node. The threshold value is used to identify the types of node in the proposed model.

DRp : Depletion power of the node

\section{Related Works}

Prasad Calyam and et all, [3] implemented a framework in Voice and Video over IP (VVOIP) with enhanced quality of experience techniques. This estimation technique helps us to identify the size estimation of network packets that are transmitted in the network. The proposed model features an offline model of QoE that is 
expressed as a function of measurable network factors. Using the GAP-model, proposed framework estimates the quality with specific grades. Jau-Yang Chang and et all, [4] implemented a Multi-hop relay network to reduce the deployment cost by deploying relay stations. Cluster based RS deployment scheme is proposed to select the appropriate location for the relay stations. Demand nodes are calculated from the distance between the transmission nodes in the cluster of nodes. Relay stations are deployed in the cluster to enhance the transmission of packets. Traffic demands and uniform cluster concepts are considered to reduce the data transmission distance of the demand nodes

A.Bieszczad and et all, [5] proposed an energy harvesting technique for remote sensor networking applications. To maximize the packet delivery ratio, transmission scheduling scheme for multi-terminal cooperative energy harvesting network is proposed. Probability of successful delivery of packets virtually has same performance as the state-of-the-art threshold based scheme. Fei Peng and et all, [6] proposed a protocol to enhance the MAC layer for application to adopt to mobile speed variation and degrade the network performance. Adaptive decision making is carried out to update the neighbor nodes in the mobile network. This approach drastically increased the performance of the network and disconnects the mobile nodes to achieve better performance. This approach enhances the power usage in mobile nodes and can increase the packet delivery performance.

\section{Honey-Bee Model}

Ad-hoc network is a collection of mobile nodes that are dynamically connected and move across the network [7]. Remote region uses sensor nodes to analyze some input, and the sensor nodes are not accessible easily. So if there is any power failure in sensor node may affect the transmission of packets, and replacement or energy recharge may not take place often. Sleep deprivation attack continuously communicates with the single sensor node to reduce the power source and depletes the entire power source to make the node as a dead node. To prevent these types of attacks, we have proposed Honey-Bee model in ad-hoc network. Nodes in the network are classified as various types as follows.

- Queen Node

- Drone Node

- Worker Node

- Dead Node

Colony represent the group of nodes with various power sources that are located in the specific area. The area determination depends upon the transmission range of nodes. Each node represents the mobile host who are capable of transmitting packets to the next node in the colony. Figure 1 represents the group of nodes combined to form a colony. Honey-bee model is composite of all nodes which include source, destination and transition nodes.

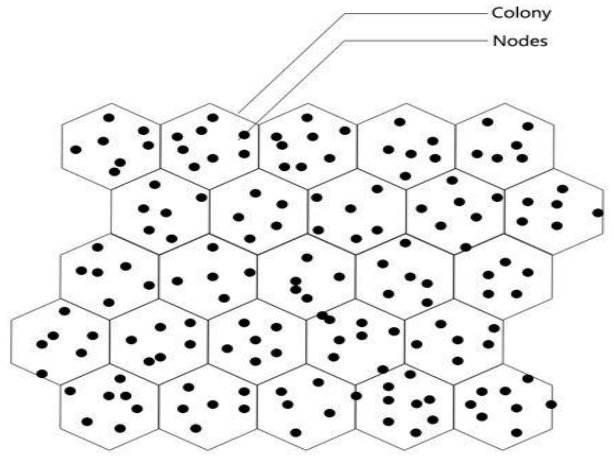

Figure 1: Honey-Bee hive model

\section{Queen Node $(Q)$}

Queen nodes are nodes, which have high power capacity that is capable of controlling the transmission of the packets. These nodes control the packet transmission over the network.Queen node is the first node to receive RREQ in the colony. It is responsible for directing RREQ to the next colony if there is no match in the routing table. Node with high power within the colony is selected as queen node as in equation 1 .

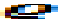

$$
=(\mathrm{Rp}>\mathrm{Tp}) \& \&(\text { Low DRp })[\text { Power } 80 \%-100 \%](1)
$$

\section{Drone Node $\left({ }^{\widetilde{\sigma}}\right)$}

Drone node receives the RREP signal from the neighboring colony. Drone maintains the routing table which gets updated during the transmission of the RREQ signal by the Queen node. Drone node maintains the power details of each node. It shares the power details to the Queen node which decides to replace the other node if the power of the node changes from the threshold value.

$$
\delta=(\mathrm{Rp}>\mathrm{Tp}) \& \&(\text { Low DRp })[\text { Power 30\% - 50\% ] }
$$

\section{Worker Node $\left(^{W}\right)$}

Worker node transmits packets to the next node in the colony. Queen node determines the routing path from drone node and initiates the transmission process. Worker node transmits packets until it finds the packet drop or another failure. Drone node maintains the power of the worker nodes and elects another node if the worker node fails to transmit.

$W^{*}=(\mathrm{Rp}>\mathrm{Tp}) \& \&($ Low DRp ) [ Power 50\% - 80\%]

\section{Delegate Node $(\wp)$}

Delegate nodes are the nodes that are ready for the replacement by the Drone node. These node details are maintained in the Drone node and are shared with the Queen node. When there is any failure in nodes, Delegate node takes responsibility to maintain the task further.

$\wp=(\mathrm{Rp}>\mathrm{Tp}) \& \&($ High/Low DRp ) [ Power above 50\%]

\section{Dead Node $(\Phi)$}

Dead nodes are the nodes which have a low-power source which cannot involve in the transmission of packets. Sometimes dead node acts as drone nodes to store only cache table.

$\boldsymbol{\Phi}=(\mathrm{Rp}<\mathrm{Tp}) \& \&($ High/Low DRp ) [ Power below 30\%]

\section{RREQ}

Table 1: RREQ Packet Transmission Header

\begin{tabular}{|l|l|l|l|l|l|l|l|l|l|}
\hline Type & $\mathrm{J}$ & $\mathrm{R}$ & $\mathrm{G}$ & $\mathrm{D}$ & $\mathrm{U}$ & SIZE & STYPE & Reserved & $\begin{array}{l}\text { HOP } \\
\text { COUNT }\end{array}$ \\
\hline & & & & & & \\
\hline
\end{tabular}

Table 1 shows the RREQ packet transmitted by the source node to determine the route [8]. We have added SIZE section in the header which carries the estimated total size of the packet that is to be transmitted. If it is live streaming packets, the size will be set as the maximum value. RREQ transmits necessary details of the size of the packet which helps to manage the power utilization for 
the worker node [9]. Depending upon the size of the packet, worker node is chosen by the queen node.

\section{RREP}

Table 2: RREP Packet Transmission Header

\begin{tabular}{|c|c|c|c|c|c|c|}
\hline Type & $\mathrm{R}$ & $\mathrm{A}$ & Reserved & SIZE & $\begin{array}{l}\text { CAN_- }_{-} \\
\text {TRANSMIT }\end{array}$ & $\begin{array}{l}\text { HOP } \\
\text { COUNT }\end{array}$ \\
\hline \multicolumn{7}{|c|}{ Destination IP Address } \\
\hline \multicolumn{7}{|c|}{ Destination Sequence Number } \\
\hline \multicolumn{7}{|c|}{ Originator IP Address } \\
\hline \multicolumn{7}{|c|}{ Lifetime } \\
\hline \multicolumn{3}{|c|}{$\begin{array}{l}\text { Drone IP } \\
\text { Address }\end{array}$} & $\begin{array}{l}\text { Worker IP } \\
\text { Address }\end{array}$ & \multicolumn{3}{|c|}{ Delegate IP Address } \\
\hline
\end{tabular}

Table 2 shows the RREP packet of proposed modification in AODV protocol [10]. SIZE slot in the header is used to identify size of the packet. CAN_TRANSMIT flag identifies the path that is used to transmit the packets over the network. If protocol determines the two different path such as worker nodes in a colony has low power and another colony with long chain of a node with high power, protocol chose the path to transmit the packet depending upon this flag. Queen node in a colony prepares RREP response with the flag set if the worker node can handle the transmission.

\section{RERR}

Table 3: RERR Packet Transmission Header

\begin{tabular}{|l|l|l|l|l|l|l|}
\hline Type & $\mathrm{R}$ & $\mathrm{A}$ & Reserved & SIZE & CAN_TRANSMIT & $\begin{array}{l}\text { HOP } \\
\text { COUNT }\end{array}$ \\
\hline & \\
\hline & Destination IP Address \\
\hline \multicolumn{5}{|l|}{ Destination Sequence Number } \\
\hline Originator IP Address \\
\hline Lifetime \\
$\begin{array}{l}\text { Drone IP } \\
\text { Address }\end{array}$ & $\begin{array}{l}\text { Worker IP } \\
\text { Address }\end{array}$ & Delegate IP Address \\
\hline
\end{tabular}

RERR request is sent when there is any break in link in one or more destination as shown in table 3. SIZE slot in the packet determines the number of size remained during the transmission failure. Depending upon the pending transmission size, queen node in the neighbor colony identifies the alternate route.

\section{Honey-Bee Formation}

Wireless network has a cluster of mobile nodes with various power resource factors. These nodes are classified under five types depending on the power consumption [11]. We have enhanced this model with the separation of each task, which reduces the computation power and reduces the power consumption. Colony is formed between the combinations of all types of a node in the area as shown in figure 2. These colonies are a piece of executing the model which handles transmission of packets and maintains routing cache.

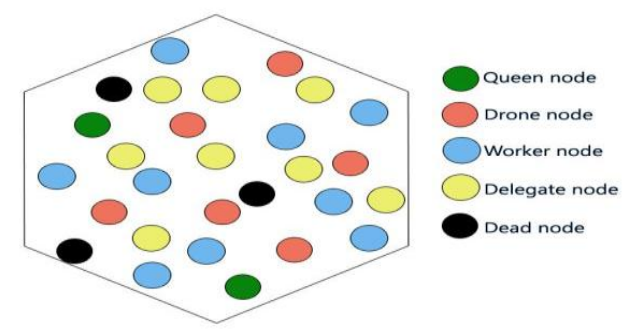

Figure 2: Honey-Bee formation

Colony can communicate to other colonies through worker node for transmission of packets and use drone node for RREQ transmission. Hexagonal representation is a virtual design to inhabit the honey bee hive model.

Rate of change of power consumed between point $\mathrm{P}$ and $\mathrm{Q}$ (slope equation),

$f(x)=(Y 2-Y 1) /(X 2-X 1)$

Total rate of change over the time limit in power consumption

$\int_{0}^{n} f(x) d x$

\section{Algorithm for Colony Formation}

Colony ColonyFormation(Nodes nodes)

Begin

Colony newColony;

$\mathrm{f}(\mathrm{x})=(\mathrm{Y} 2-\mathrm{Y} 1) /(\mathrm{X} 2-\mathrm{X} 1)$

PowerConsumptionValue $=\int_{0}^{n} f(x) d x$ newColony.OverallPowerConsumption=PowerConsumptionValue; optimalValue $=$ PowerConsumptionValue $/$ no.of.nodes $*$ average TotalPower

For each Node in nodes

Begin

If (Node.RemainingPower > optimalValue \&\&

Node.RemainingPower $>$ percentage (80) \& \&

Node.RemainingPower $<$ percentage $(100) \& \&$

Node.PowerConsumptionValue $<0.5$ )

Begin

newColony.AddQueenNode(Node );

End

Else If (Node.RemainingPower > optimalValue $\& \&$

Node.RemainingPower>percentage (30) \& \&

Node.RemainingPower $<$ percentage (50) $\& \&$

Node.PowerConsumptionValue $<0.5$ )

Begin

newColony.AddDroneNode(Node );

End

Else If (Node.RemainingPower > optimalValue $\& \&$

Node.RemainingPower>percentage (50) \&\&

Node.RemainingPower $<$ percentage (80) \&\&

Node.PowerConsumptionValue $<0.5$ )

Begin

newColony.AddWorkerNode(Node );

End

Else If (Node.RemainingPower > optimalValue \&\&

Node.RemainingPower $<$ percentage (50)

$\& \&$ Node.PowerConsumptionValue $<0.5$ )

Begin

newColony.AddDelegateNode(Node );

End

Else

newColony.AddDeadNode(Node );

Return newColony;

End

\section{Hierarchical Clustering Method}

Hierarchical clustering is a method of clustering based on the hierarchical strategy [12]. The linkage determines the distance between sets of observations as a function of the pair wise distances between observations as in figure 3, 4 and 5. Based on the mean linkage value, the nodes are grouped into clusters. Clusters are built with the hierarchy from the individual elements by progressively merging clusters.

Single-linkage clustering, $C s=\min \{d(a, b): a \in A, b \in B\}$ (8) 


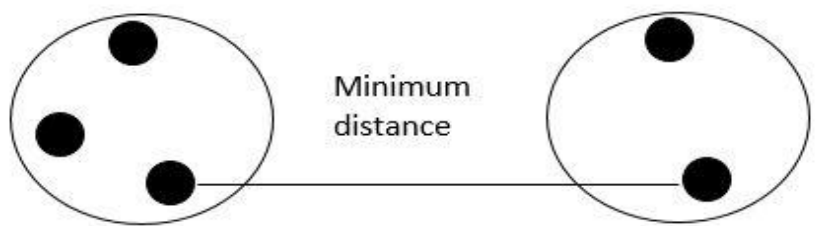

Figure 3: Single-linkage clustering

Complete-linkage clustering, $\mathrm{Cc}=\max \{d(a, b): a \in A, b \in B\}$

(9)

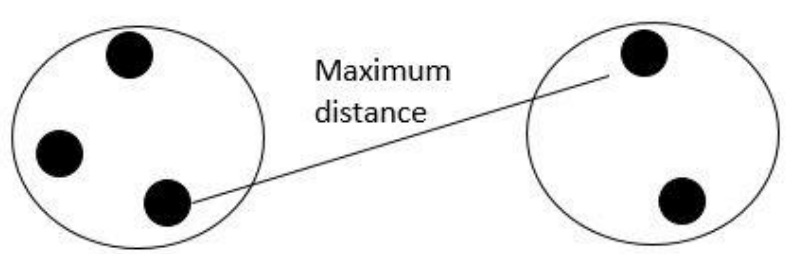

Figure 4: Complete-linkage clustering

Mean or average linkage clustering, Cmean $=\frac{1}{|A||| B \mid} \sum_{a \in A} \sum_{b \in B} d(a, b)$

(10)

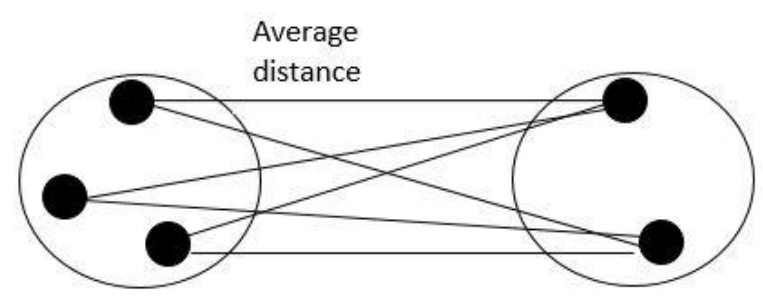

Figure 5: Complete-linkage clustering

\section{Routing Cache in Drone Node}

Drone node maintains a cache of routing table with RREP request [13] of neighbor colony nodes as shown in table 4. This information is used to identify the effective route to send packets without raising RREQ requests. Since size of the packets is used to analyze the transmission node, distinct routes are used for different size of the packets.

Table 4: Routing Cache

\begin{tabular}{l|l} 
Packet Max Size & Route Path
\end{tabular}

During the real-time transmission or streaming of the packets, the highest size on the routing table is considered for the route identification phase as in table 4 . When the queen node identifies the RREQ-ACK request, the routing table is updated against the size of the packet that can be handled through the given route.

\section{Cluster Formation and Illustration}

Manhattan distance is used to determine the distance between the nodes in the cluster $[14,15]$. Figure 6 shows the number of nodes in the network. Each node is mobile and has similar transmission range.

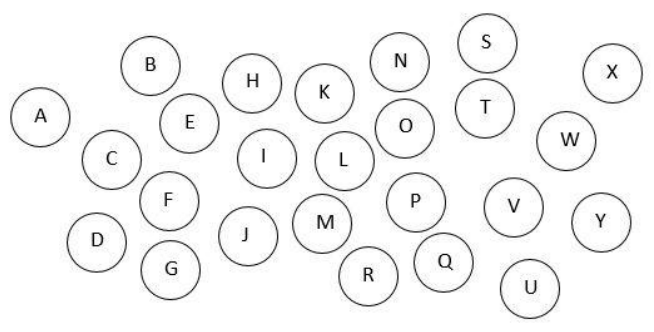

Figure 6: Cluster of nodes
Manhattan distance $=|| a-b||_{i}=\sum_{i}\left|a_{i}-b_{i}\right|$

Distance matrix is used to identify the distance between nodes and used for the construction of the clusters. Let $\mathrm{n}$ be the number of nodes, then $\mathrm{N} \mathrm{X} \mathrm{N}$ matrix is required to store the distance of all the matrixes. Below table shows the distance matrix between the node $\mathrm{A}, \mathrm{B}, \mathrm{C}, \mathrm{D}$, and $\mathrm{E}$.

Table 5: NXN Matrix for Manhattan Distance

\begin{tabular}{|l|l|l|l|l|l|}
\hline Nodes & A & B & C & D & E \\
\hline A & 0 & 165 & 129 & 244 & 211 \\
\hline B & 165 & 0 & 122 & 201 & 90 \\
\hline C & 129 & 122 & 0 & 144 & 142 \\
\hline D & 244 & 201 & 144 & 0 & 213 \\
\hline E & 211 & 90 & 142 & 213 & 0 \\
\hline
\end{tabular}

Based on the distance matrix as shown in table 5, the nodes are clustered based upon the transmission range. Nodes are clustered, which fall in the same transmission range. Figure 7 shows the nodes collectively grouped into many clusters and these cluster are responsible for transmitting packets.

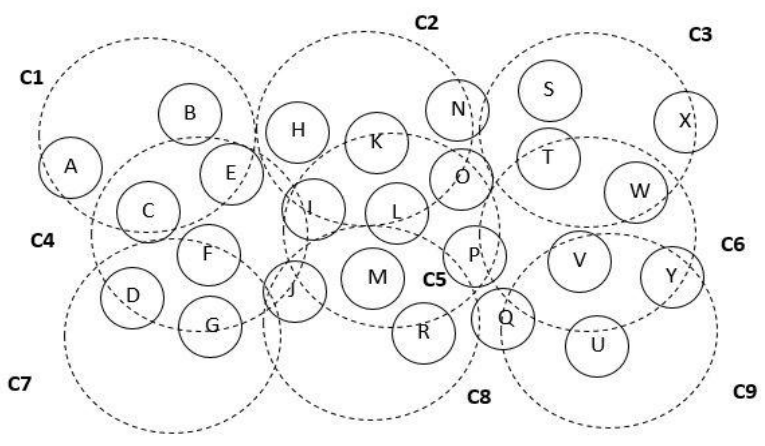

Figure 7: Cluster formation from nodes

A node in cluster $\mathrm{C} 1$ need to send packets of size $\mathrm{N}$ to the node in the cluster C8. Proposed AODV sends RREQ signal to the cluster C5 through Queen Node. Drone node in the cluster C1 holds the routing table regarding the path to the cluster C8. Queen Node checks the routing table in the drone node to identify the routing path to the destination against the size $\mathrm{N}$. If there is no routing path, the queen node initiates the RREQ request to the next queen node of neighbour cluster $\mathrm{C} 5$ as shown in figure 8. And RREQ request is sent to the next cluster $\mathrm{C} 3, \mathrm{C} 4, \mathrm{C} 6, \mathrm{C} 7$ and $\mathrm{C} 9$ as shown in figure 9. Again routing table is cross checked in the drone node on each clusters and if there is no routing path is identified, the request is transferred to the next queen node. Size of the packet that is transmitted in the RREQ signal is used as weigh factor for the routing path. If the power source in the cluster has minimal value that cannot handle enormous size of the packets, alternate route is carried out by the queen node. After successful identification of the routing path, the drone node in each cluster is updated and transferred to the source node in cluster $\mathrm{C} 1$.

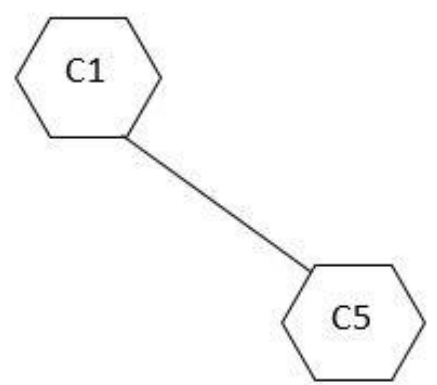

Figure 8: Initial stage of RREQ transmission. 


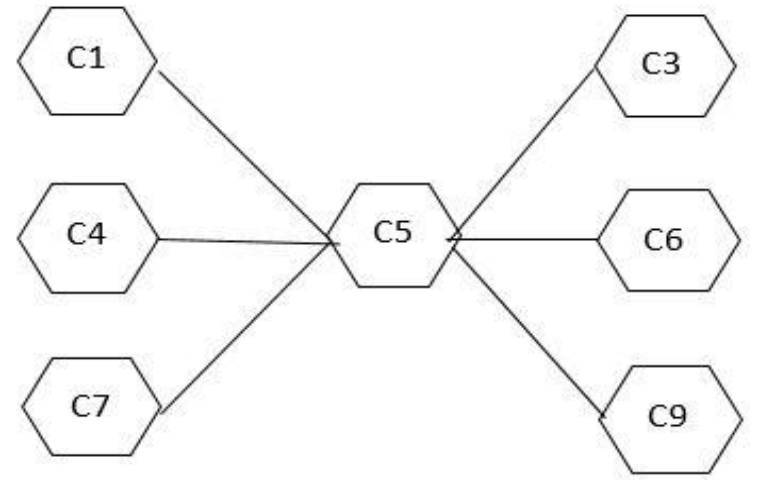

Figure 9: RRE from cluster C5 to nodes C4,C7,C3,C6 and C9

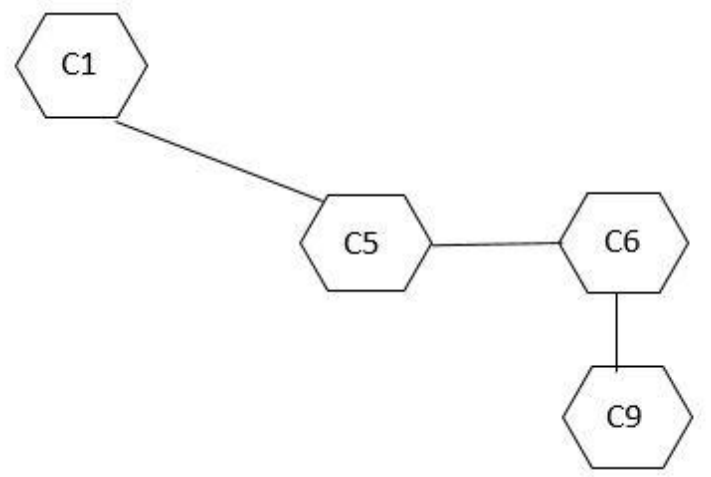

Figure 10: Overall transmission of RREQ from $\mathrm{C} 1$ to cluster C9.

When RREQ request hits the cluster C6 from cluster $\mathrm{C} 3$, the RREQ node navigate to the cluster C5. Since AODV internally handles the sequence number mechanism, the RREQ request is rejected to avoid the dead-lock.

RREP-ACK returns five different paths such as $(\mathrm{C} 1, \mathrm{C} 5, \mathrm{C} 4, \mathrm{C} 7)$, (C1,C5,C7,C8), (C1,C5,C3,C6,C9,C8), (C1,C5,C6,C9,C8) and $(\mathrm{C} 1, \mathrm{C} 5, \mathrm{C} 9, \mathrm{C} 8)$. Queen node compares the health of each path returned from RREQ-ACK. Size $\mathrm{N}$ is used to identify the appropriate path to transfer the packet. Queen node assign the task to the worker node to start transmitting the packets. Queen node in the cluster elects a worker node in each cluster to transfer the packet to the destination. Figure 10 shows the overall transmission path of the packets which is identified as the efficient path.

\section{Results and Discussion}

Random graphs are used to identify the probability distribution over the graphs using edge probability technique. We have used four other routing protocols such as Power Aware Routing Protocol [17], Dynamic Source Routing [18], Flow-State extensions to Dynamic Source Routing [19] and AODV to compare with the proposed honey-bee method. We used network simulator to simulate the scenario with various models and results are explained in graphs [20,21, 22, and 23].

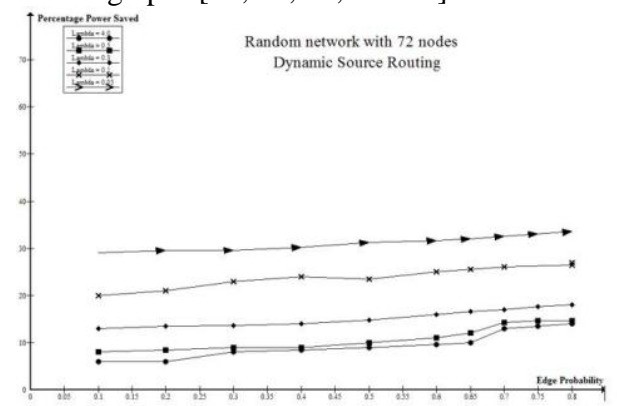

Figure 11: Edge probability graph for DSR

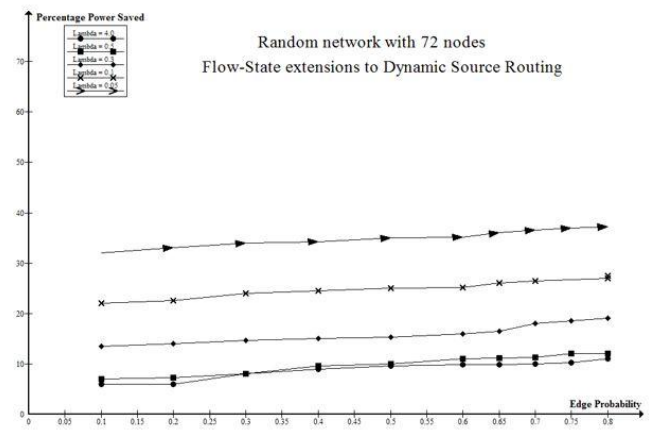

Figure 12: Edge probability graph for Flow-state to DSR

Figure 11, 12, 13, 14, 15 and 16 shows the random network comparison graph of major protocols. The lambda value is set to 0.25 for the edge probability. Power saved during the transmission of packets [24] is higher in the proposed honey-bee model. It shows that Dynamic source protocol saves power when the edge probability is higher compared to AODV. Enhanced AODV with honey-bee model performs high during high edge probability due to the distributive nature of the model. When sleep depreciation is encountered, Queen Node in the cluster elects other worker node if any of the node is identified as low power unit. If cluster cannot handle the requested size of transmission, other transmission path is sent to the source to handle transmission. This helps to avoid the node shutdown due to the power loss [25].

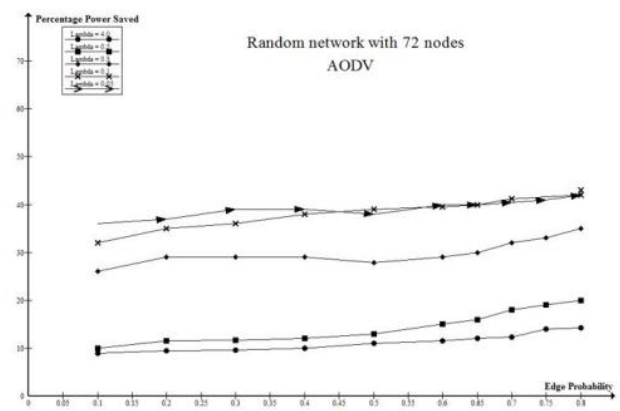

Figure 13: Edge probability graph for AODV

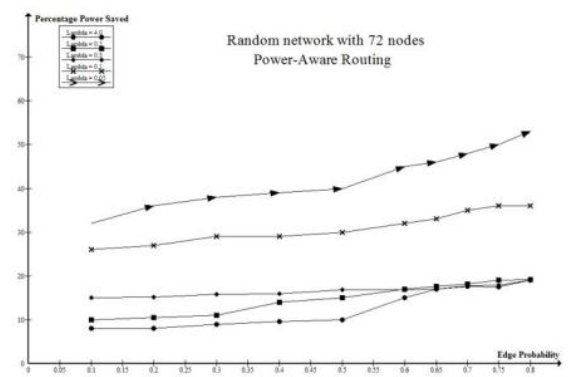

Figure 14: Edge probability graph for Power aware routing

Power-aware routing technique involved in power centric routing method to enhance the power consumption of the nodes in mobile network. Percentage of power saved is higher compared to other routing protocols

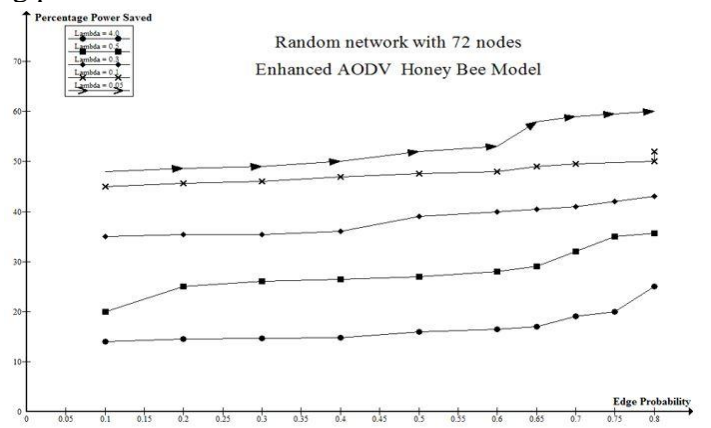

Figure 15: Edge probability graph for enhanced AODV honey bee model 
It is interesting to observe that the power savings are greater in larger networks because larger networks have more routes [20]. Power saving increases with packet load since packets are transmitted on various paths.

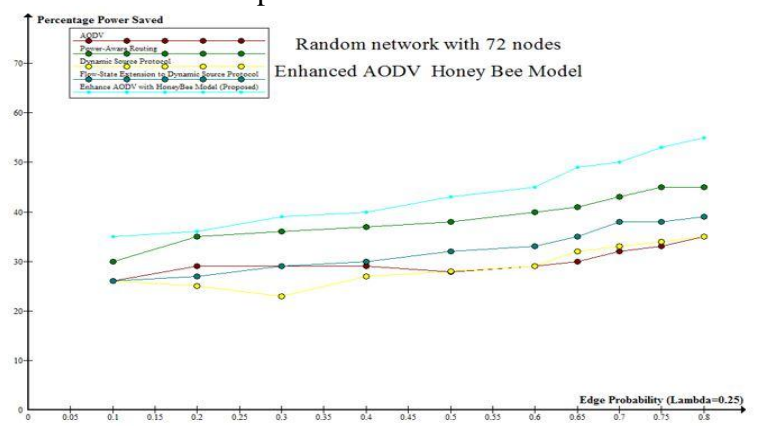

Figure 16: Comparative graph for Edge probability graph

\section{Conclusion}

Honey-bee cluster formation divides the work load among the nodes in the network. These computed power consumption technique distributes workload among the nodes which reduces the power consumption on single node. Sensor nodes are placed in remote location with limited energy source. The power cannot be replaced easily when it is depleted [26]. Transmission of packets concentrated on only one node may deplete the power source. Sleep deprivation attack may deplete the power fully which results in removal of node from the network. Proposed model efficiently allocate transmission task to nodes based on power-elector method. Hence nodes involve only on some part of transmission which enhance the power saving mechanism. Comparison result over the protocols shows the efficiency of the honey-bee model and defines the power consumption over the network efficiently. Each type of nodes in the honey-bee model defines the overall performance of the model. Since power plays a vital role in the honey-bee model, power replaced mechanism should be harvested as energy harvesting technique [27]. In future, energy harvesting model over the proposed model should be defined to enhance the power play model.

\section{References}

[1] Palaniappan S \& Chellan K, "Energy-efficient stable routing using QoS monitoring agents in MANET", EURASIP Journal on Wireless Communications and Networking, (2015).

[2] Madhurikkha S \& Sabitha R, "An Efficient Integral PowerElector Method with Enhanced AODV to Avoid Sleep Deprivation in Manet", Indian Journal of Science and Technology, (2016).

[3] Calyam P, Ekici E, Lee CG, Haffner M \& Howes N, "A "GAPModel" based framework for online VVoIP QoE measurement", Journal of Communications and Networks, Vol.9, No.4,(2006), pp.446-456.

[4] Chang JY \& Chen YW, "A cluster-based relay station deployment scheme for multi-hop relay networks", Journal of Communications and networks, Vol.17, No.1,(2015), pp.84-92.

[5] Pagurek BB \& White T, "An adaptive scheduling scheme for cooperative energy harvesting networks", Journal of Communications and Networks, Vol.17, No.3, (2015).

[6] Peng F, Peng B \& Leung VC, "An application oriented power saving MAC protocol for wireless sensor networks", Wireless Personal Communications, Vol.67, No.2,(2012), pp.279-293.

[7] Chang JY \& Ju PH, "An efficient cluster-based power saving scheme for wireless sensor networks", EURASIP Journal on Wireless Communications and Networking, (2012).

[8] Zhou Y, Huang Z, Wang J, Huang R \& Yu D, “An energyefficient random verification protocol for the detection of node clone attacks in wireless sensor networks", EURASIP Journal on Wireless Communications and Networking, (2014).

[9] Nie N \& Comaniciu C, "Energy efficient AODV routing in CDMA ad hoc networks using beamforming", EURASIP Journal on Wireless Communications and Networking, (2006).
[10] Kanakaris V, Ndzi DL, Ovaliadis K \& Yang Y, "A new RREQ message forwarding technique based on Bayesian probability theory", EURASIP Journal on Wireless Communications and Networking, (2012).

[11] Abbas NI, Ilkan M \& Ozen E, "Fuzzy approach to improving route stability of the AODV routing protocol", EURASIP Journal on Wireless Communications and Networking, (2015).

[12] Cheng ST, Shih JS, Chang TY, Horng GJ \& Chou CL, "MLPAconservation mechanism in wireless sensor network environments", EURASIP Journal on Wireless Communications and Networking, (2012).

[13] Smail O, Cousin B, Mekki R \& Mekkakia Z, "A multipath energyconserving routing protocol for wireless ad hoc networks lifetime improvement", EURASIP Journal on Wireless Communications and Networking, (2014).

[14] Jin Y, Xu J \& Qiu L, "Energy-efficient scheduling with individual packet delay constraints and non-ideal circuit power", Journal of Communications and Networks, Vol.16, No.1,(2014), pp.36-44.

[15] Sarkar M \& Roy DB, "Prevention of sleep deprivation attacks using clustering", 3rd International Conference on Electronics Computer Technology (ICECT), Vol.5, (2011), pp.391-394.

[16] Hamad S, Radhi N \& Al-Raweshidy H, "Candidate Neighbours to Rebroadcast the RREQ for efficient flooding in mobile ad hoc network", Wireless Advanced (WiAd), (2011), pp.26-29.

[17] Wu J, Ren S, Jiang Y \& Song L, "QoS-aware multihop routing in wireless sensor networks with power control using demodulationand-forward protocol", EURASI Journal on Wireless Communications and Networking, (2012).

[18] Sharma AK, Sharma RK \& Jain V, "An efficient power aware routing protocol for mobile adhoc networks using cluster head", 3rd International Conference on Computing for Sustainable Global Development (INDIACom), (2016), pp.2955-2958.

[19] Yang H, Tian C, Cheng B, Ci L, Wang Z \& Yang M, "CDS-A Collaborative Dynamic Source Routing Protocol for Wireless Sensor Network", Ubiquitous Intelligence and Computing and IEEE 12th Intl Conf on Autonomic and Trusted Computing and IEEE 15th Intl Conf on Scalable Computing and Communications and Its Associated Workshops (UIC-ATC-ScalCom), (2015), pp. 617-621.

[20] Ns-2 network simulator, 2008, http://www.isi.edu/nsnam/ns/

[21] CMU Monarch BPensions to NS-2, 2008

http://www.monarch.cs.cmu.edu/cmu-ns.html

[22] Marc Greis' Tutorial for the UCB/LBNL/VINT Network Simulator "ns", 2008

http://web.uct.ac.za/depts/commnetwork/tutorial_ns_full.pdf

[23] Installing OLSR on NS-2 , 2008.

http://masimum.inf.um.es/um-olsr/html

[24] Li S, Xiao S, Zhang M \& Zhang X, "Power saving and improving the throughput of spectrum sharing in wideband cognitive radio networks", Journal of Communications and Networks, Vol.17, No.4,(2015), pp.394-405.

[25] Yen HH, Lee SS \& Yap FG, "A novel optimization-based approach for minimum power multicast in wireless networks", Journal of Communications and Networks, Vol.13, No.1,(2011), pp.26-31.

[26] Quang PTA \& Kim DS, "Clustering algorithm of hierarchical structures in large-scale wireless sensor and actuator networks", Journal of Communications and Networks, Vol.17, No.5,(2015), pp.473-481.

[27] Shin J \& Suh C, "CREEC: Chain routing with even energy consumption", Journal of Communications and Networks, Vol.13, No.1,(2011), pp.17-25. 